\title{
Efficacy and safety of mecobalamin combined with vestibular rehabilitation training for acute vestibular neuritis: a systematic review and meta-analysis
}

\author{
Xiaohua Sun ${ }^{1,2 \#}$, Xin $\mathrm{Li}^{3,4 \#}$, Dong Yang ${ }^{5}$ \\ ${ }^{1}$ Department of Rehabilitation, Beijing Tsinghua Changgung Hospital, Beijing, China; ${ }^{2}$ School of Sport Medicine and Rehabilitation, Beijing \\ Sport University, Beijing, China; ${ }^{3}$ Department of Otorhinolaryngology, Beijing Tsinghua Changgung Hospital, Beijing, China; ${ }^{4}$ School of Clinical \\ Medicine, Tsinghua University, Beijing, China; ${ }^{5}$ Department of Otorhinolaryngology, Tianjin Medical University General Hospital, Tianjin, China \\ Contributions: (I) Conception and design: X Sun, X Li; (II) Administrative support: D Yang; (III) Provision of study materials or patients: X Sun, D \\ Yang; (IV) Collection and assembly of data: All authors; (V) Data analysis and interpretation: All authors; (VI) Manuscript writing: All authors; (VII) \\ Final approval of manuscript: All authors. \\ "These authors contributed equally to this work. \\ Correspondence to: Xin Li. No. 168, Litang Road, Changping District, Beijing 102218, China. Email: entlixintsinghua@sina.com.
}

Background: This study aimed to investigate the efficacy and safety of mecobalamin combined with vestibular rehabilitation training in acute vestibular neuritis and to improve the clinical therapeutic effect in vestibular nerve disease.

Methods: We performed a literature search of the PubMed, Medline, China National Knowledge Infrastructure (CNKI), and other databases from the date of establishment of the database until the present. The search terms included "mecobalamin", "vestibular rehabilitation training", "vestibular rehabilitation therapy", and "vestibular neuritis". References of the comparative study of vestibular rehabilitation training and vestibular rehabilitation training combined with mecobalamin were screened. Boolean logic retrieval was adopted, and Review Manager software was employed.

Results: Meta-analysis was conducted on a total of four studies with a low risk of bias. The activities specific balance confidence scale $(\mathrm{ABC})$ scores of the two groups were heterogeneous $\left(\mathrm{Chi}^{2}=8.56, \mathrm{I}^{2}=88 \%\right.$, $\mathrm{P}=0.003)$, and a fixed-effect model (FEM) analysis indicated that there were no significant differences in the $\mathrm{ABC}$ between the groups after treatment $(\mathrm{Z}=0.67, \mathrm{P}=0.50)$. It may be that mecobalamin combined with vestibular rehabilitation training effectively alleviated the symptoms of vestibular neuritis in the experimental group, thereby reducing the canal paresis $(\mathrm{CP})$ value. In addition, there was no heterogeneous dizziness handicap inventory (DHI) between the groups after treatment $\left(\mathrm{Chi}^{2}=20.75, \mathrm{I}^{2}=86 \%, \mathrm{P}=0.0001\right)$; finite element method (FEM) analysis showed that the DHI of the experimental group after 6 months of treatment was notably lower compared to that of the control group $(\mathrm{Z}=3.20, \mathrm{P}=0.001)$.

Discussion: Mecobalamin combined with vestibular rehabilitation training can effectively improve vertigo and other symptoms of acute vestibular neuritis patients, with high effectiveness and safety.

Keywords: Mecobalamin; vestibular rehabilitation training; vestibular neuritis; vertigo

Submitted Sep 27, 2021. Accepted for publication Nov 18, 2021.

doi: 10.21037/apm-21-3171

View this article at: https://dx.doi.org/10.21037/apm-21-3171

(c) Annals of Palliative Medicine. All rights reserved. 


\section{Introduction}

Vestibular neuritis is a sudden vertigo disorder caused by acute damage to one of the vestibular nerves. When inflammation of the anterior vestibular nerve occurs, the balance model generated by the inner ear and the position information generated by the head cannot be normally transmitted to the brain, leading to acute or persistent vertigo in patients (1). Patients in the acute and convalescent phases exhibit different clinical symptoms; the acute phase manifests as persistent and severe dizziness, accompanied by nausea, vomiting, and other symptoms. During the recovery period, the vertigo symptoms of patients disappear, and at this time, the condition manifests as non-rotating dizziness, instability, and temporary vertigo after head movement. Also, patients can walk independently and deviate to one side, and the direction of deviation is related to the state of vestibular compensation $(2,3)$. The most common cause of vestibular neuritis is viral infection of the vestibular nerve (measles, influenza, herpes zoster, varicella virus, etc.). Other causes include ischemia (acute ischemia of the vestibular and labyrinthian nerves) and bacterial infection $(4,5)$. Treatment of vestibular neuritis includes specific treatment (glucocorticoids, antiviral therapy, etc.), symptomatic treatment, and vestibular rehabilitation training in the acute phase (6). In the acute phase, hormones (methylprednisolone, prednisone, etc.) are generally chosen to improve vestibular function, and antiviral therapy is required for viral infection $(7,8)$. Mecobalamin, also known as methyl vitamin B12, is a neurotrophic drug. It can provide necessary substances for nerve repair by promoting the synthesis of protein, phosphoric acid, and phospholipid, and has a good therapeutic effect on vestibular neuritis and other diseases (9). After usage of mecobalamin, adverse reactions such as headache, fever, sweating, rash, blood pressure drop, and dyspnea may occur, and timely treatment measures should be taken when adverse reactions occur. For patients with vestibular neuritis, in addition to taking mecobalamin, diphenhydramine can also be used for sedation and hydrogen binding effect. In addition, it can relieve symptoms and patients' discomfort.

Vestibular rehabilitation therapy (VRT) is a nonpharmacological, non-traumatic, and physical therapy for the treatment of disorders of central and peripheral balance $(10,11)$. Patients with vestibular dysfunction were treated with train-based therapy to improve their ability to coordinate and control balance by vestibular position, vision and proprioception, and to mobilize the compensatory function of the central nervous system
$(12,13)$. Vestibular rehabilitation training can accelerate the recovery of patients with acute vestibular neuritis and reduce the disability degree of patients with permanent vestibular injury. It has a certain positive effect on the longterm recovery of patients as well as the recovery of balance function $(14,15)$. Training methods mainly include fixation stability visual tracking training, static and dynamic posture stability training, and functional activity training (16).

Clinical studies found that the proportion of patients with acute vestibular neuritis returning to normal after treatment is about $60 \%$, and the recurrence rate is high. Patients with vestibular neuritis have a high risk of recurrence of vertigo symptoms and are often accompanied by refractory benign paroxysmal positional vertigo (17). Therefore, we performed a meta-analysis to systematically evaluate the efficacy and safety of mecobalamin combined with vestibular rehabilitation training for acute vestibular neuritis. We present the following article in accordance with the PRISMA reporting checklist (available at https://apm.amegroups.com/ article/view/10.21037/apm-21-3171/rc).

\section{Methods}

\section{Literature search}

Documents were searched using Boolean logic retrieval. We performed an electronic literature search of the PubMed, Medline, Embase, Chinese Biomedical Literature Database, CNKI, and Google Scholar databases. The search terms included "mecobalamin", "vestibular rehabilitation training", "vestibular rehabilitation therapy", and "vestibular neuritis". The list of references for all included reviews was traced to find references that were not indexed. The retrieval time was up to October 30, 2020, and evaluation was performed using RevMan 5.2 in the Cochrane system. A variety of terms were searched in free combination, multiple retrieval was performed to determine the literature, and the literature trace was recorded by the search engine. In addition, contact was made with researchers to ascertain the most recent research progress.

\section{Inclusion and exclusion criteria}

The included articles were required to meet all of the following criteria: (I) studies involving patients with confirmed acute vestibular neuritis without pathological type restriction; (II) intervention measures for patients in the control group were vestibular rehabilitation training (including static balance connection, vestibular eye reflex 
training, and vestibular neck reflex training) without mecobalamin intervention; (III) patients in experimental group received mecobalamin intervention combined with vestibular rehabilitation training; and (IV) randomized clinical trials (RCTs), as well as prospective cohort and casecontrol studies.

Literature was excluded according to the following criteria: (I) studies involving subjects with serious infectious diseases; (II) literature without randomized controlled trials (RCTs); (III) articles without valid data or missing literature; (IV) cases involving overlapped data or subjects; and (V) repeatedly published articles and those with small sample selections.

After three pre-experiments, two experts independently read the titles, abstracts, and full texts. Disagreements were resolved by consultation or arbitration by a third expert. Other disagreements were also addressed according to the method mentioned above.

\section{Quality assessment}

The Newcastle-Ottawa Scale (NOS) from the Cochrane Collaboration was used to assess the pathological control studies. The subjects' results, case comparisons, and intergroup comparisons were evaluated by the star system, with nine stars representing a full score. Seven stars or above was considered as high quality; that is, a low risk of bias. Meanwhile, articles with $\leq$ one star were considered low quality (high bias), and studies with two-six stars were considered medium quality (medium bias). After three pre-experiments, two experts independently assessed the article quality.

\section{Data extraction}

Using a unified Excel table, the experts independently extracted the following data after three pre-experiments: (I) the first author and the year of publication; (II) the case numbers of the two groups; (III) grouping and intervention of the two groups; and (IV) recovery indicators, such as activities-specific balance confidence (ABC) scale and the Dizziness Handicap Inventory (DHI) score, before and after treatment.

\section{Statistical methods}

In Review manager 5.3, the mean difference (MD) and 95\%
CI (confidence interval) indicated efficacy analysis statistics for continuity variables. The heterogeneity test ( $Q$ test) was performed initially, followed by the risk bias evaluation using the bias risk assessment map in the software. Each effect was expressed with a $95 \%$ CI. A fixed effects model (FEM) was employed if $\mathrm{P}>0.1$ and $\mathrm{I}^{2}<50 \%$; otherwise, a random effects model (REM) was used for processing.

\section{Results}

\section{Summary of literature collection results and NOS scale rating}

As shown in Figure 1, 526 references were obtained. Of these, 226 were excluded after reading the abstracts and titles of the articles, and 296 were excluded after reading the full texts of the articles. Finally, four references were included. The excluded articles involved subjects with primary acute and chronic cardiopulmonary dysfunction and mental diseases (100 articles), animal experiments (44 articles), repetitive subjects (36 articles), missing data (50 articles), no hematopoietic stem cell transplantation or killer cell therapy (40 articles), and lack of original data (26 articles). Table 1 shows the major data, covering the period from 2005 to 2020. The NOS scale rating is illustrated in Figure 2; $25 \%$ of the included studies had $\geq$ seven stars, $50 \%$ had two-six stars, and $25 \%$ had >two stars, all of which were over medium quality.

\section{Risk of bias of the included literatures}

Figures 3,4 display the multiple risk bias evaluation results. RevMan was used to generate the bias risk assessment maps for each methodological feature included in the literature. Studies with random sequence generation (selection), assignment concealment (selection), blinding of outcome evaluation (measurement), incomplete data (follow-up), and selective reporting (reporting) were considered to be very low risk of bias. As for those with blinding bias (implementation) and other biases of both subjects and researchers are approximately $25 \%$. The other literatures had a very low risk of bias, except for the references of Micarelli [2019] and Micarelli [2017].

\section{DHI scores of the two groups of patients before treatment}

Figure 5 shows the DHI scores of two groups before treatment. The research results of Micarelli [2019] occupy 
Identification of studies via databases and registers
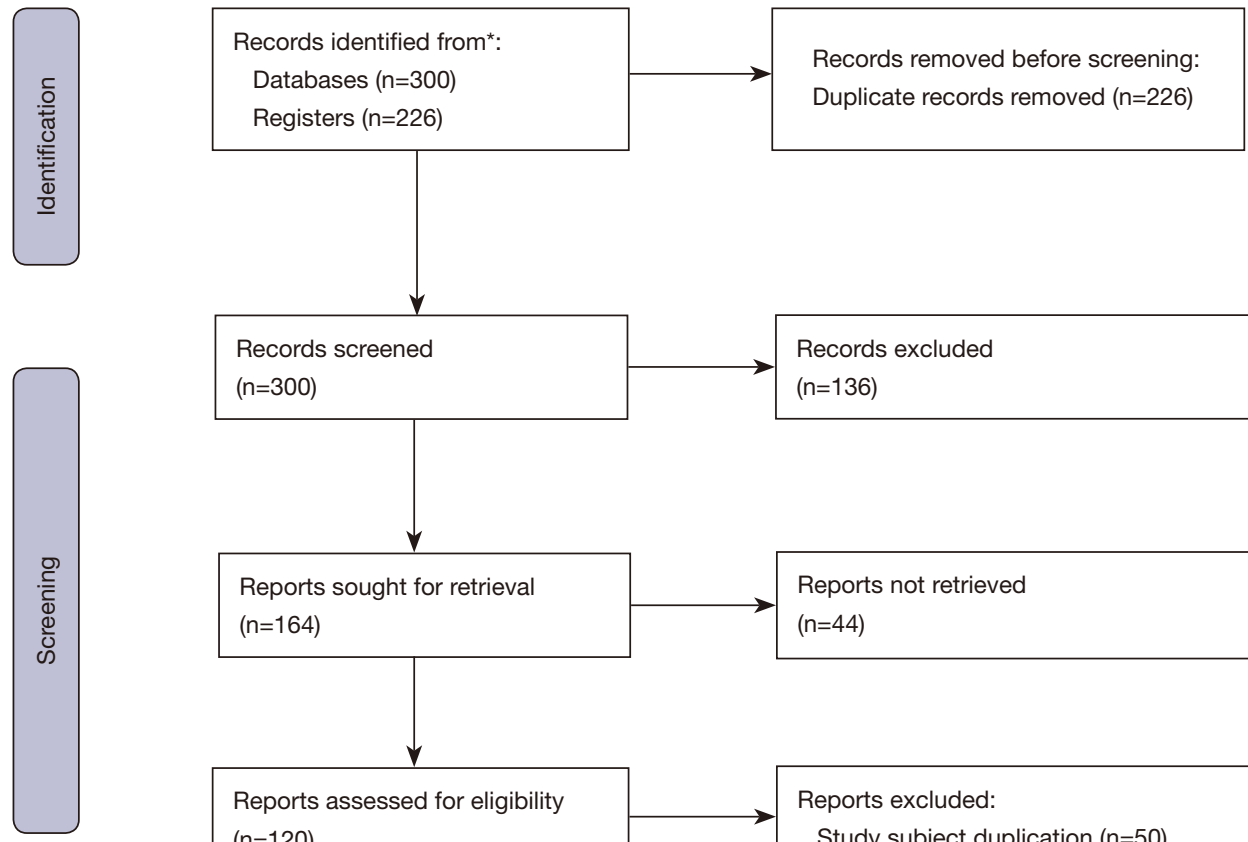

Reports not retrieved

$(n=44)$

Reports assessed for eligibility $(n=120)$

Reports excluded:

Study subject duplication $(n=50)$

The relevant information of the

research can't be extracted $(n=66)$

Studies included in review

$(n=4)$

Figure 1 Screening process. *, databases and registers.

Table 1 Basic data of the included studies

\begin{tabular}{|c|c|c|c|c|c|c|c|}
\hline First author & $\begin{array}{c}\text { Year of } \\
\text { publication }\end{array}$ & \multicolumn{2}{|c|}{ Case numbers } & \multicolumn{2}{|c|}{ Age } & Means of intervention & $\begin{array}{l}\text { Disease duration } \\
\text { (month) }\end{array}$ \\
\hline Micarelli (9) & 2017 & 24 & 23 & $49.72 \pm 10.34$ & $50.48 \pm 9.12$ & $\begin{array}{c}\text { Medication combined with } \\
\text { vestibular rehabilitation training }\end{array}$ & $9.37 \pm 1.55$ \\
\hline Micarelli (10) & 2019 & 24 & 23 & $74.3 \pm 4.7$ & $76.9 \pm 4.7$ & $\begin{array}{l}\text { Medication combined with } \\
\text { vestibular rehabilitation training }\end{array}$ & $23.5 \pm 5$ \\
\hline Krueger (11) & 2011 & 25 & 25 & $60.1 \pm 5.44$ & $60.0 \pm 3$ & $\begin{array}{c}\text { Medication combined with } \\
\text { vestibular rehabilitation training }\end{array}$ & $29.2 \pm 40.6$ \\
\hline Garcia (12) & 2013 & 23 & 21 & $47.9 \pm 19$ & $47.65 \pm 20$ & $\begin{array}{l}\text { Medication combined with } \\
\text { vestibular rehabilitation training }\end{array}$ & $4-48$ \\
\hline
\end{tabular}




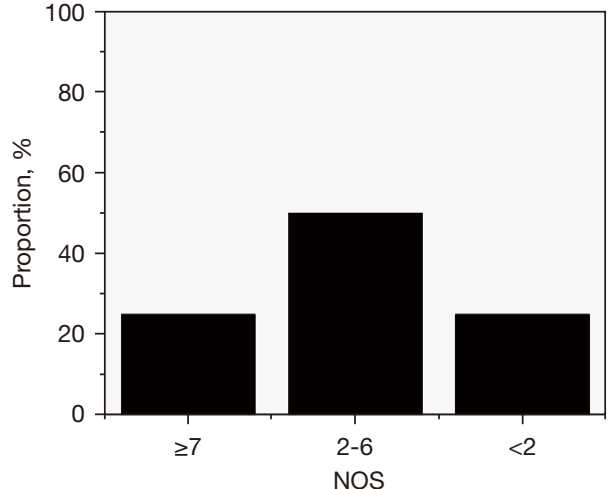

Figure 2 NOS scale for quality rating. NOS, Newcastle-Ottawa Scale. the highest percentage (81.9\%), followed by Micarelli [2017] (6.5\%) and Garcia [2013] (6.5\%). In addition, the horizontal lines (HL) of the $95 \%$ CIs of most studies crossed the invalid vertical line (IVL). There were 96 control cases and 92 experimental cases in the four included articles. No heterogeneity was indicated in the DHI scores between the two groups before treatment $\left(\mathrm{Chi}^{2}=2.76, \mathrm{I}^{2}=0 \%\right.$, $\mathrm{P}=0.43)$. The combined effect size, which is represented by a diamond block, crossed the IVL, and the Odds ratio (OR) value was 2.64 (95\% CI: -0.58 to 5.86). FEM analysis indicated no considerable differences in the DHI scores between the groups before treatment $(\mathrm{Z}=1.61, \mathrm{P}=0.11)$.

On both sides of the invalid midline are the circles, as

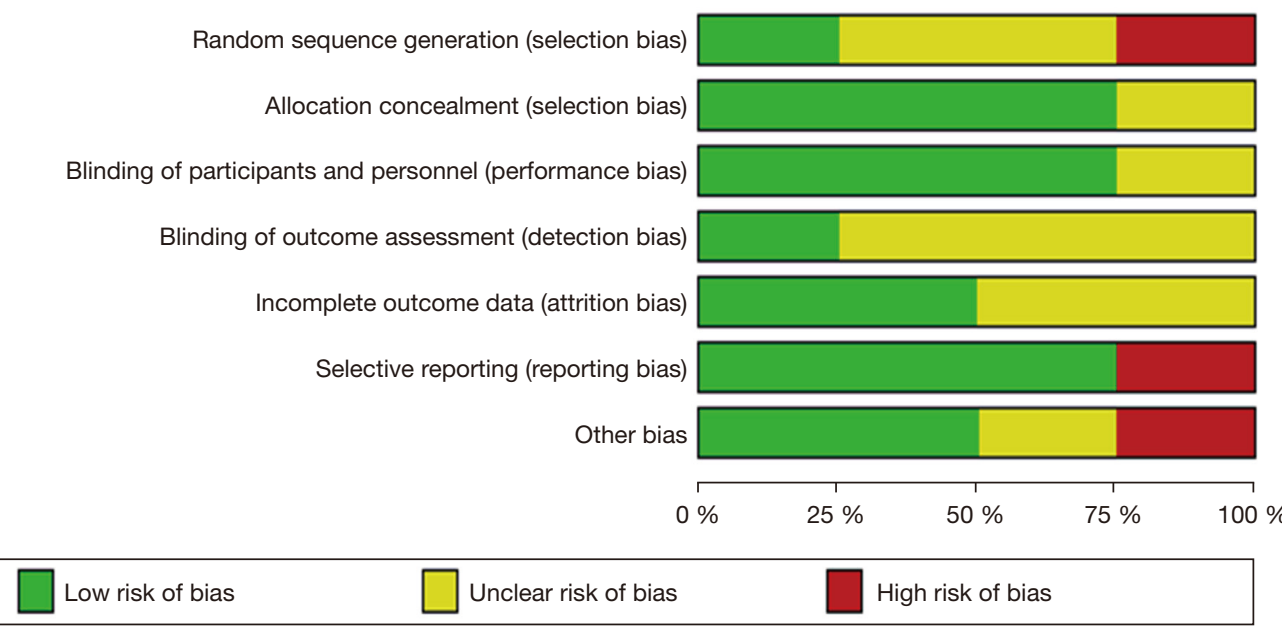

Figure 3 Risk of bias assessment.

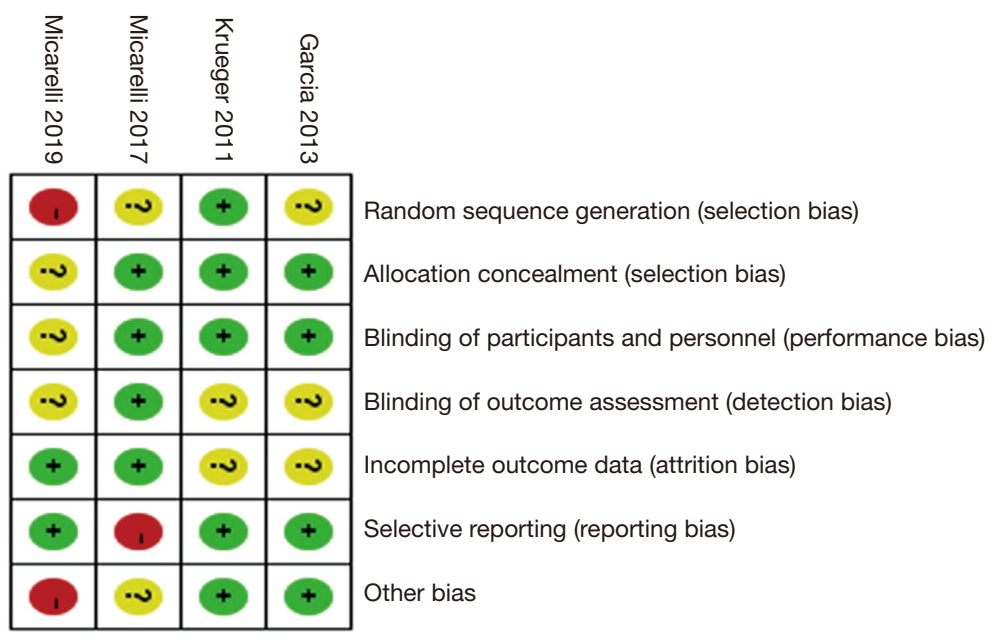

Figure 4 Evaluation results of multiple risk bias. 


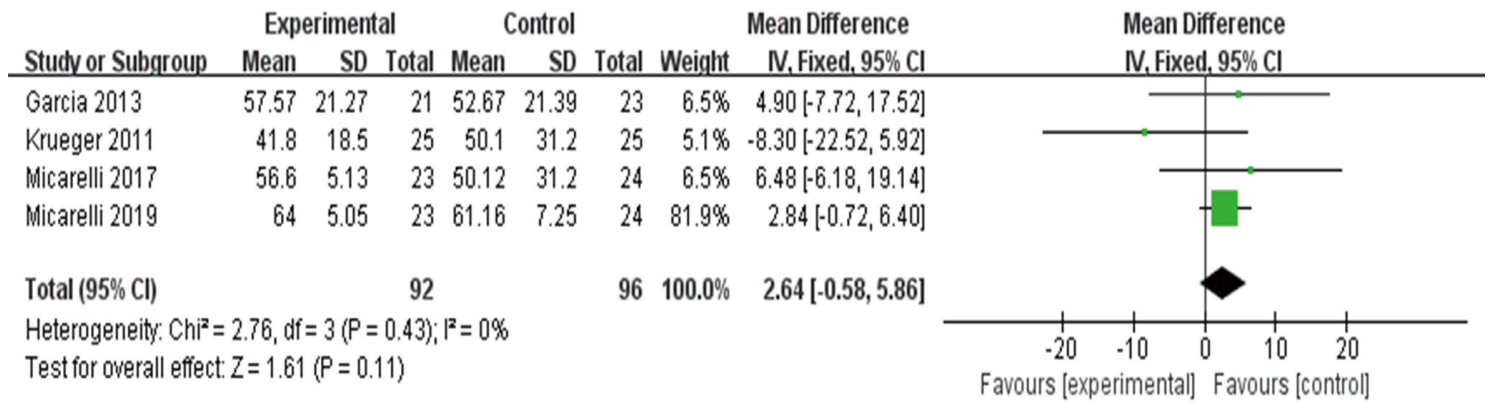

Figure 5 Comparison of DHI before treatment. DHI, Dizziness Handicap Inventory.

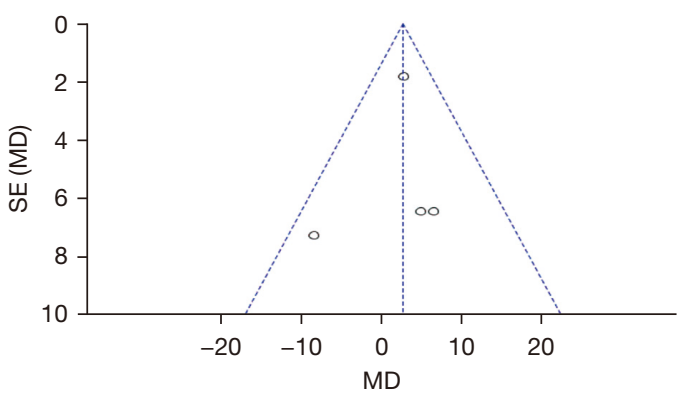

Figure 6 Funnel chart illustrating the DHI of the patients before treatment. DHI, Dizziness Handicap Inventory.

presented in Figure 6, which are uniform and symmetrical, indicating no obvious publication bias.

\section{DHI scores of the two groups of patients after treatment}

Figure 7 shows the DHI scores after 6 months of treatment. The results of Micarelli [2017] account for the highest percentage (33.0\%), followed by those of Micarelli [2019] (32.4\%). In addition, most $95 \%$ CI's HL fell to the left of the IVL, and only few research HLs crossed the IVL. Among the four studies, there were 96 subjects in the control group and 92 subjects in the experimental group. There was no heterogeneous DHI between the groups after treatment $\left(\mathrm{Chi}^{2}=20.75, \mathrm{I}^{2}=86 \%, \mathrm{P}=0.0001\right)$. The diamond block was on the left of the IVL, and the OR value was -10.56 (95\% CI: -17.03 to -4.09$)$. FEM analysis showed that the DHI of the experimental group after six months of treatment was remarkably lower compared to the control group $(\mathrm{Z}=3.20$, $\mathrm{P}=0.001)$.

Figure 8 shows the DHI scores of the two groups after treatment. The circles are in the top area, indicating high accuracy. The circles are on both sides of the midline, which are symmetrical, signifying no obvious publication bias.

\section{Comparison of the ABC scores between the two groups before treatment}

Figure 9 shows the $\mathrm{ABC}$ scores of the two groups before treatment. The results of Micarelli [2019] occupied the highest percentage $(51.9 \%)$. In addition, most of $95 \%$ CI's HLs were on the left of the IVL, and the research HL crossed the IVL. There were 48 subjects were in the control group and 46 cases in the experimental group. No statistical heterogeneity was suggested in the $\mathrm{ABC}$ scores of the two groups before treatment $\left(\mathrm{Chi}^{2}=0.83, \mathrm{I}^{2}=0 \%, \mathrm{P}=0.36\right)$. The diamond block was on the right side of the IVL, and the OR value was -1.37 (95\% CI: -3.59 to 0.85 ). FEM analysis indicated no considerable differences in the $\mathrm{ABC}$ scores between the groups before treatment $(\mathrm{Z}=1.21, \mathrm{P}=0.23)$.

Figure 10 shows that the circles are in the top area, indicating high accuracy. The circles are symmetrical, signifying no obvious publication bias.

\section{Comparison of the ABC scores between the groups after treatment}

Figure 11 is a comparison of the $\mathrm{ABC}$ scores of patients after 6 months of treatment. Micarelli's [2019] results accounted for the highest percentage (50.1\%). In addition, most $95 \%$ CI's HLs were on the right of the IVL, and the research HL did not cross the IVL. Among the included four studies, there were 48 controls and 46 experimental subjects. The $\mathrm{ABC}$ scores of two groups were heterogeneous $\left(\mathrm{Chi}^{2}=8.56\right.$, $\left.\mathrm{I}^{2}=88 \%, \mathrm{P}=0.003\right)$. The diamond was on the right of the IVL, and the OR value was 2.14 (95\% CI: -4.13 to 8.42 ). FEM analysis indicated no considerable differences in the $\mathrm{ABC}$ scores between the groups after treatment $(\mathrm{Z}=0.67$, $\mathrm{P}=0.50)$.

Figure 12 shows that the circles are on both sides of the midline and are symmetrical, suggesting no obvious 


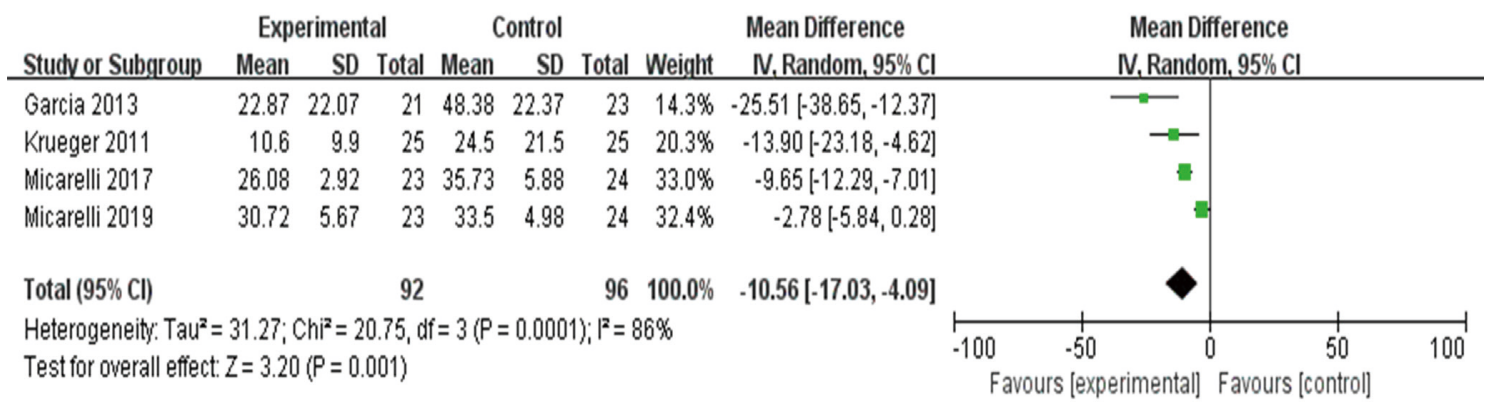

Figure 7 DHI scores of patients after treatment. DHI, Dizziness Handicap Inventory.

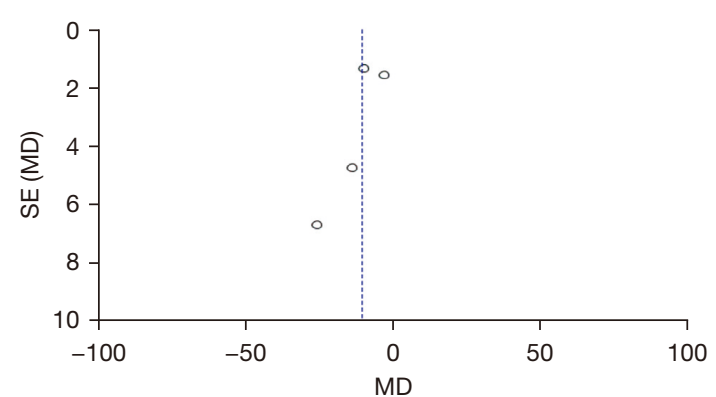

Figure 8 Funnel chart of the DHI of the patients after treatment. DHI, Dizziness Handicap Inventory.

publication bias.

\section{Discussion}

When inflammation of the anterior vestibular nerve occurs, the balance model generated by the inner ear and the position information generated by the head cannot be normally transmitted to the brain, leading to acute or persistent vertigo in patients, which is a sudden vertigo disorder caused by acute damage to one of the vestibular nerves. Patients in the acute and convalescent phases of vestibular neuritis exhibit different clinical symptoms. The acute phase manifests as persistent and severe dizziness, accompanied by nausea, vomiting, and other symptoms. During recovery, the vertigo symptoms of patients disappear, and at this time, the condition manifests as nonrotating dizziness, instability, and temporary vertigo after head movement. The patient can walk independently and deviate to one side, and the direction of deviation is related to the state of vestibular compensation. The most common cause of vestibular neuritis is viral infection of the vestibular nerve, including measles, influenza, shingles, and varicella.
The vestibular system has a strong compensatory ability.

The theoretical basis of vestibular functional rehabilitation is the brain's reintegration of visual, vestibular, and sensory systems, which strengthen the function of the visual proprioceptive system and replace the damaged part of vestibular function, thereby achieving a new balance (18). Amber et al. [2012] (19) found that the central nervous system of the human body has plasticity and adaptability to vestibular function damage. Through repeated training, the body can adapt to the asymmetrical information of the external anterior court. Vestibular training primarily helps patients improve their balance stability or visual compensatory ability through vestibular adaptation and substitution formed by long-term stimulation. The most common cause of vestibular neuritis is viral infection of the vestibular nerve (e.g., measles, influenza, shingles, varicella, etc.), while other causes include ischemia (acute ischemia of the vestibular and labyrinth nerves) or bacterial infection. In the acute stage of vestibular neuritis, the otolithostone and semicircular canal function of patients are simultaneously damaged. The addition of medication to conventional treatment can significantly improve the clinical prognosis of patients with acute preacoustic neuritis. The treatment of vestibular neuritis includes specific treatments (e.g., glucocorticoids, antiviral therapy, etc.) in the acute phase, symptomatic treatment, and vestibular rehabilitation training.

Among the 4 included literatures, nine were randomized controlled and grouped, and only one was a retrospective analysis, which introduced bias into the study; however, it had little influence on the results of the study. It was unstable to analyze single samples, so quantitative synthesis of each included article was implemented via metaanalysis, and sampling errors from different populations were avoided. Moreover, in terms of sample size, different 


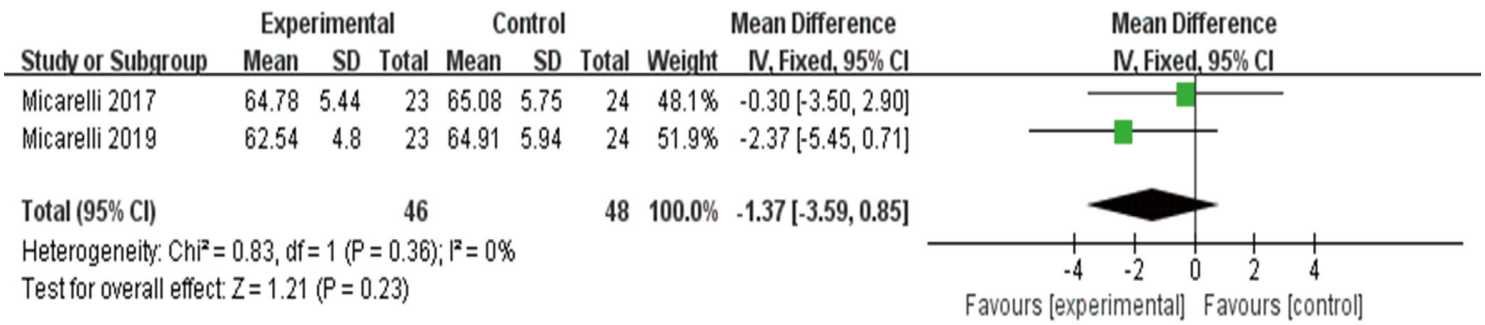

Figure 9 Comparison of the $\mathrm{ABC}$ scores between the groups before treatment. ABC, activities-specific balance confidence.

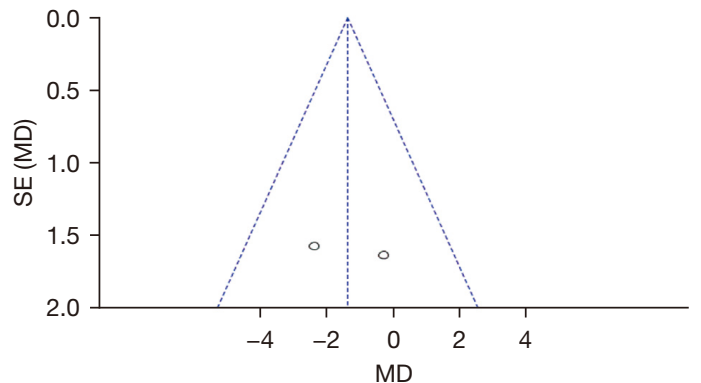

Figure 10 Funnel chart of the $\mathrm{ABC}$ scores before treatment. $\mathrm{ABC}$, activities-specific balance confidence.

weights were assigned to the corresponding results. In this way, the sample size was expanded, and reliability of conclusion was guaranteed. The number of articles included in this study was limited, and thus, a large sample size is required to reduce bias.

Four comparative studies of vestibular rehabilitation training and vestibular rehabilitation training combined with mecobalamin were meta-analyzed, which were retrieved according to the Boolean composite logic method, in order to investigate the efficacy and safety of mecobalamin combined with vestibular rehabilitation training for acute vestibular neuritis. The $\mathrm{ABC}$ scores of two groups were heterogeneous $\left(\mathrm{Chi}^{2}=8.56, \mathrm{I}^{2}=88 \%, \mathrm{P}=0.003\right)$, and FEM analysis indicated no considerable differences in the $\mathrm{ABC}$ scores between the groups after treatment. It may be that mecobalamin combined with vestibular rehabilitation training effectively alleviated the symptoms of vestibular neuritis in the experimental group, thereby reducing the canal paresis $(\mathrm{CP})$ value. In addition, there was no heterogeneity in the DHI scores between the groups after treatment $\left(\mathrm{Chi}^{2}=20.75, \mathrm{I}^{2}=86 \%, \mathrm{P}=0.0001\right)$, and FEM analysis showed that the DHI scores of the experimental group after 6 months of treatment was notably lower than that of the control group $(\mathrm{Z}=3.20, \mathrm{P}=0.001)$. These results are similar to the findings of Tramontano et al. [2018] (20), indicating that mecobalamin combined with vestibular rehabilitation training can effectively improve vertigo and other symptoms in patients, with high effectiveness and safety. It was also found that $60 \%$ of patients returned to normal vestibular function after treatment, and the recurrence rate was high. Moreover, patients with cured vestibular neuritis exhibited a high risk of recurrence of vertigo symptoms, which are often accompanied by refractory benign paroxygenic positional vertigo. Vestibular rehabilitation training can accelerate the rehabilitation of patients with acute vestibular neuritis and reduce the disability degree of patients with permanent vestibular injury. It has a certain positive effect on the long-term recovery of patients and the recovery of balance function. Therefore, training should be performed, including fixation stability visual tracking training, static and dynamic posture stability training, and functional activity training.

\section{Conclusions}

In this study, meta-analysis is carried out on four comparative studies of vestibular rehabilitation training and vestibular rehabilitation training combined with mecobalamin, which were retrieved according to the Boolean composite logic method, in order to investigate the efficacy and safety of mecobalamin combined with vestibular rehabilitation training for acute vestibular neuritis. The results showed that mecobalamin combined with vestibular rehabilitation training could effectively improve the vertigo and other symptoms of patients, with high effectiveness and safety. However, confounding factors resulted in bias. The references selected were all case controls, and thus, survival bias existed. Moreover, multiple risk factors and indicators were not discussed, which affected the pooled effect size. Patients with acute vestibular neuritis will be considered in future research, so as to explore the impact of joint 


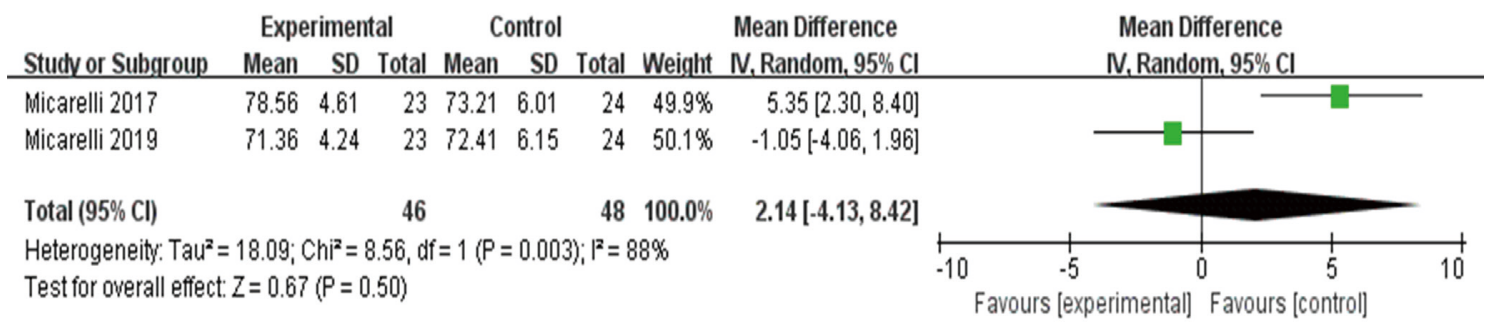

Figure 11 Comparison of the $\mathrm{ABC}$ scores. $\mathrm{ABC}$, activities-specific balance confidence.

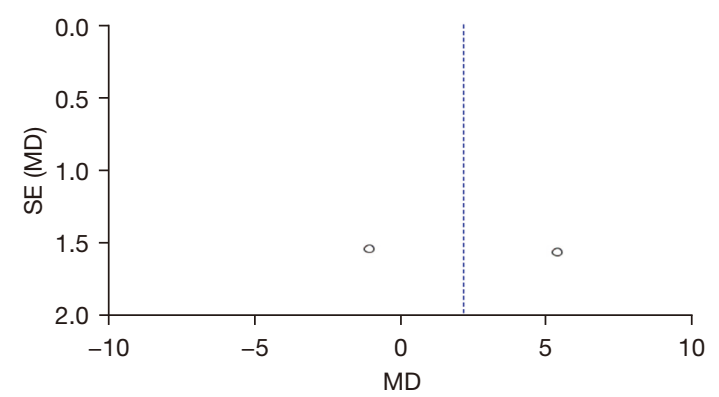

Figure 12 Funnel plot of the ABC scores after treatment. ABC, activities-specific balance confidence.

intervention in patients with acute vestibular neuritis. In conclusion, this research provides a treatment reference and related data for vestibular neuritis and other diseases.

\section{Acknowledgments}

Funding: This study was supported by the Beijing Municipal Administration of Hospitals in Cubating Program, code PX2016031.

\section{Footnote}

Reporting Checklist: The authors have completed the PRISMA reporting checklist. Available at https://apm. amegroups.com/article/view/10.21037/apm-21-3171/rc

Conflicts of Interest: All authors have completed the ICMJE uniform disclosure form (available at https://apm. amegroups.com/article/view/10.21037/apm-21-3171/coif). The authors have no conflicts of interest to declare.

Ethical Statement: The authors are accountable for all aspects of the work in ensuring that questions related to the accuracy or integrity of any part of the work are appropriately investigated and resolved.

Open Access Statement: This is an Open Access article distributed in accordance with the Creative Commons Attribution-NonCommercial-NoDerivs 4.0 International License (CC BY-NC-ND 4.0), which permits the noncommercial replication and distribution of the article with the strict proviso that no changes or edits are made and the original work is properly cited (including links to both the formal publication through the relevant DOI and the license). See: https://creativecommons.org/licenses/by-nc-nd/4.0/.

\section{References}

1. Barkay O, Niv E, Santo E, et al. Low-dose heparin for the prevention of post-ERCP pancreatitis: a randomized placebo-controlled trial. Surg Endosc 2008;22:1971-6.

2. Domínguez MO. Treatment and rehabilitation in vestibular neuritis. Rev Laryngol Otol Rhinol (Bord) 2005;126:283-6.

3. Fishman JM, Burgess C, Waddell A. Corticosteroids for the treatment of idiopathic acute vestibular dysfunction (vestibular neuritis). Cochrane Database Syst Rev 2011;(5):CD008607.

4. Johns P, Quinn J. Clinical diagnosis of benign paroxysmal positional vertigo and vestibular neuritis. CMAJ 2020;192:E182-6.

5. Le TN, Westerberg BD, Lea J. Vestibular Neuritis: Recent Advances in Etiology, Diagnostic Evaluation, and Treatment. Adv Otorhinolaryngol 2019;82:87-92.

6. Martin-Sanz E, Rueda A, Esteban-Sanchez J, et al. Vestibular Restoration and Adaptation in Vestibular Neuritis and Ramsay Hunt Syndrome With Vertigo. Otol Neurotol 2017;38:e203-8.

7. Roberts RA. Management of Recurrent Vestibular Neuritis in a Patient Treated for Rheumatoid Arthritis. Am J Audiol 2018;27:19-24. 
8. Strupp M, Zingler VC, Arbusow V, et al.

Methylprednisolone, valacyclovir, or the combination for vestibular neuritis. N Engl J Med 2004;351:354-61.

9. Micarelli A, Viziano A, Augimeri I, et al. Threedimensional head-mounted gaming task procedure maximizes effects of vestibular rehabilitation in unilateral vestibular hypofunction: a randomized controlled pilot trial. Int J Rehabil Res 2017;40:325-32.

10. Micarelli A, Viziano A, Micarelli B, et al. Vestibular rehabilitation in older adults with and without mild cognitive impairment: Effects of virtual reality using a head-mounted display. Arch Gerontol Geriatr 2019;83:246-56.

11. Krueger WW. Controlling motion sickness and spatial disorientation and enhancing vestibular rehabilitation with a user-worn see-through display. Laryngoscope 2011;121 Suppl 2:S17-35.

12. Garcia AP, Ganança MM, Cusin FS, et al. Vestibular rehabilitation with virtual reality in Ménière's disease. Braz J Otorhinolaryngol 2013;79:366-74.

13. Goudakos JK, Markou KD, Franco-Vidal V, et al. Corticosteroids in the treatment of vestibular neuritis: a systematic review and meta-analysis. Otol Neurotol 2010;31:183-9.

14. Fishman JM. Corticosteroids effective in idiopathic facial nerve palsy (Bell's palsy) but not necessarily in idiopathic

Cite this article as: Sun X, Li X, Yang D. Efficacy and safety of mecobalamin combined with vestibular rehabilitation training for acute vestibular neuritis: a systematic review and metaanalysis. Ann Palliat Med 2022;11(2):480-489. doi: 10.21037/ apm-21-3171 acute vestibular dysfunction (vestibular neuritis).

Laryngoscope 2011;121:2494-5.

15. Adamec I, Krbot Skorić M, Gabelić T, et al. Intravenous dexamethasone in acute management of vestibular neuritis: a randomized, placebo-controlled, single-blind trial. Eur J Emerg Med 2016;23:363-9.

16. Vroomen P. Methylprednisolone, valacyclovir, or both for vestibular neuritis. N Engl J Med. 2004;351:2344-5; author reply 2344-5.

17. Strupp M, Arbusow V, Brandt T. Exercise and drug therapy alter recovery from labyrinth lesion in humans. Ann N Y Acad Sci 2001;942:79-94.

18. Lee MY, Hyun JH, Suh MW, et al. Treatment of peripheral vestibular dysfunction using photobiomodulation. J Biomed Opt 2017;22:1-7.

19. Amber KT, Castaño JE, Angeli SI. Prophylactic valacyclovir in a patient with recurrent vestibular disturbances secondary to vestibular neuritis. Am J Otolaryngol 2012;33:487-8.

20. Tramontano M, Bergamini E, Iosa M, et al. Vestibular rehabilitation training in patients with subacute stroke: A preliminary randomized controlled trial. NeuroRehabilitation 2018;43:247-54.

(English Language Editor: A. Kassem) 